Methodology Report

\title{
Epidemiological Study of the Incidence of Cancers Eligible for Proton or Carbon Ions Therapy: Methodology and Results of Recruitment Estimation
}

\author{
Stéphanie Patin, ${ }^{1}$ Pascal Pommier, ${ }^{2}$ Hu Yi, ${ }^{1}$ Marie Hélène Baron, ${ }^{3}$ and Jacques Balosso ${ }^{1,4}$ \\ ${ }^{1}$ GCS ETOILE, 60 Avenue Rockefeller, 69008 Lyon, France \\ ${ }^{2}$ Centre Régional de Lutte Contre le Cancer Léon Bérard, 28 Rue Laennec, 69008 Lyon, France \\ ${ }^{3}$ CHU Jean Minjoz, 25030 Besançon Cedex, France \\ ${ }^{4}$ Université Joseph Fourier, Grenoble1, BP 53-38041, Grenoble Cedex 9, France
}

Correspondence should be addressed to Stéphanie Patin; stephanie.patin@centre-etoile.org

Received 19 February 2013; Revised 16 May 2013; Accepted 22 May 2013

Academic Editor: Lynnette Ferguson

Copyright (c) 2013 Stéphanie Patin et al. This is an open access article distributed under the Creative Commons Attribution License, which permits unrestricted use, distribution, and reproduction in any medium, provided the original work is properly cited.

\begin{abstract}
Context. Hadrontherapy is an innovative form of radiotherapy using beams of protons or carbon ions able to destroy some radioresistant tumours. Because these tumours are highly specific amongst all cancerous tumours, it is impossible to determine the incidence of these diseases from surveillance registries. Goal. To assess, within the Rhône-Alpes region, the incidence of cancers being hadrontherapy indications. Method. Prospective, multicentre continuous data collection during 1 year, by practitioners participating to multidisciplinary tumor board. Tumours are inoperable, radio resistant, at primary stage of development, or locally recurrent, with low metastatic potential. Results. Study involved 27 healthcare centres, 52 groups of specialist practitioners. The estimated incidence of cancers eligible for hadrontherapy in the Rhône-Alpes region in 2010, that is, for 34 locations in all, is of 8.5/100 000 inhabitants. Appraisal of the low potential of metastatic progression is impeded, because these are rare diseases, whose outcome is unfamiliar to investigators. Conclusion. Future epidemiological studies will need to focus on prognosis and on the metastatic progression rate of these diseases. Indeed, there are few information available on this subject in the literature that could be used to improve preventive measures, medical care, and the surveillance of these rare cancers.
\end{abstract}

\section{Scientific Context}

1.1. Hadrontherapy. Hadrontherapy is an innovative form of radiotherapy, based on high-technology equipment using proton or carbon ions beams to destroy tumours $[1,2]$. This treatment method enables significantly higher ballistic precision to be achieved, compared to photons (X-rays) with, as expected therapeutic benefit, an improvement of quality of life and chances of recovery [3]. Carbon ions are also specifically characterised by superior biological efficacy (relative biological effectiveness from 1.5 to 3), overcoming the radiation resistance of certain cancers to photons and even protons. Indeed, carbon ion beams when compared to $\mathrm{X}$-rays represent a distinct advantage for the treatment of highly radiation-resistant tumours [4].
1.2. Hadrontherapy Epidemiological Studies. An initial study assessing recruitment potential for proton therapy was conducted in 1998 in Italy, showing an incidence of 10825 cases/year [5]. One year later, a second study was carried out in the context of the MedAustron project for the construction of a carbon ions therapy centre in Austria. Considering patients living in Austria and neighbouring countries, the patient recruitment potential (proton and carbon) was estimated with an incidence of 13145 cases/year [6]. In France, in 2002, a third study was conducted in the context of the ETOILE medical project. This study revealed an annual incidence of approximately 5320 cases/year for carbon ions [7]. Concomitantly, a fourth study was conducted, again in the context of the MedAustron project, estimating the incidence of cases eligible for hadrontherapy in Austria at 


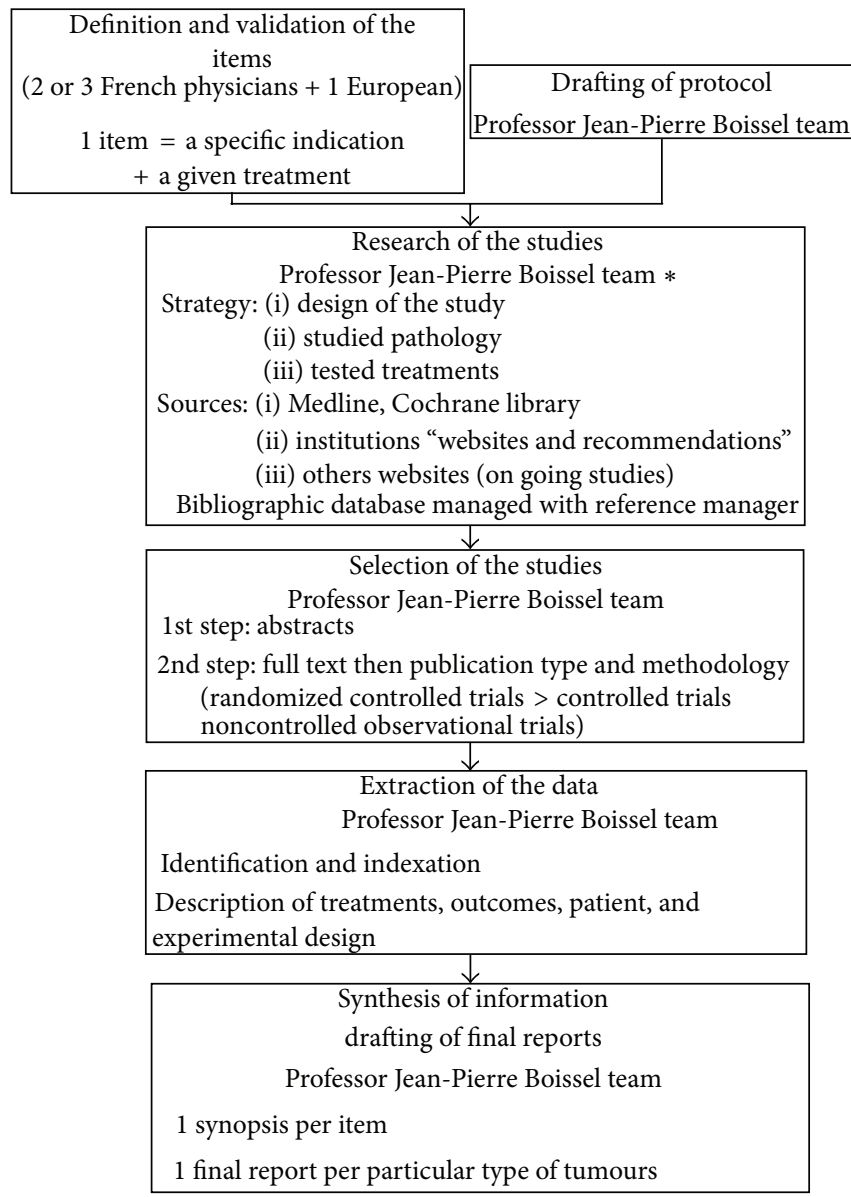

(1) Selection of all items to study. Each item is a couple of indication and treatment (example: cancer of cavum - treatment by radiochemotherapy). Relevant clinical outcomes and duration of followup were also defined.

(2) Exhaustive literature research for each item.

(3) Selection of trials.

(i) First selection based on the title and the abstract.

(ii) Second selection and after a full paper reading.

(4) Description of each selected trial.

(i) Description of patient characteristics, treatments, and toxicities.

(ii) Results on efficacy.

(5) Synthesis of efficacy results.

(6) Drafting of final reports.

(i) Methodology of research

(ii) A synopsis by item.

(iii) Detailed tables with all included studies.

(iv) References of included studies.

* Professor Jean Pierre Boissel, center of data treatment, UMR 5558 Medicine University of Laennec, Lyon, France

FIGURE 1: Literature review method.

2044 cases/year [8]. Finally, again in 2002, in the context of the project for the construction of the CNAO centre in Italy, an epidemiological study was conducted, estimating the incidence of cases eligible for carbon therapy at 3694 cases/year and proton therapy at 1885 cases/year. [9]. Since 2004, no other epidemiological studies have been published for hadrontherapy.

In 2011, however, the Japanese team from the National Institute for Radiological Sciences (NIRS), pioneer in carbon therapy, published the results of its 1994 to 2011 clinical activity. Patients were included in clinical trials and the number of patients receiving carbon therapy was 6157 [10]. This value is the incidence of treated cases, over 17 years without any notion of recruitment potential and with a rather slow ramp up of the activity.

1.3. Therapeutic Indications for Hadrontherapy. A list of priority indications that can be treated by hadrontherapy (Table 1) was drawn up by the GCS ETOILE medical group, in collaboration with national and international experts, based on published data ranked using an "evidence-based medicine" type analysis (Figure 1). This literature review was based on provided or expected medical service criteria (survival and quality of life). According to the classification used by the European Survey Group of Rare Diseases [11], the eligible tumours that can be treated by hadrontherapy conform to the criteria of rare diseases based on the following thresholds of epidemiological indicators: prevalence $\leq 50$ cases $/ 100000$ persons within a given population and incidence $\leq 6$ cases/100 000 persons/year. Indeed, for all tumor types identified during the previous hadrontherapy epidemiological studies [5-8], the calculated and cumulative incidences are all below these thresholds (Table 2). Amongst the hadrontherapy indications, 25 are tumours listed in the ORPHANET international database of rare diseases [12] (cf. ORPHANET number in Table 1).

1.4. Problem. The exact incidence of each disease eligible for hadrontherapy is difficult to determine from the available resources. Indeed, the published data [5-13] pertain to series of patients for which the aim was not to assess the level of demand for treatment. These were rather series of patients who had received the therapy, but whose recruitment had not been accounted for in tumour registries. Considering the scarcity and specific nature of the tumours concerned by hadrontherapy, it is difficult to extrapolate and assess the treatment demand from these results. Hadrontherapy indications are defined according to anatomical location of 


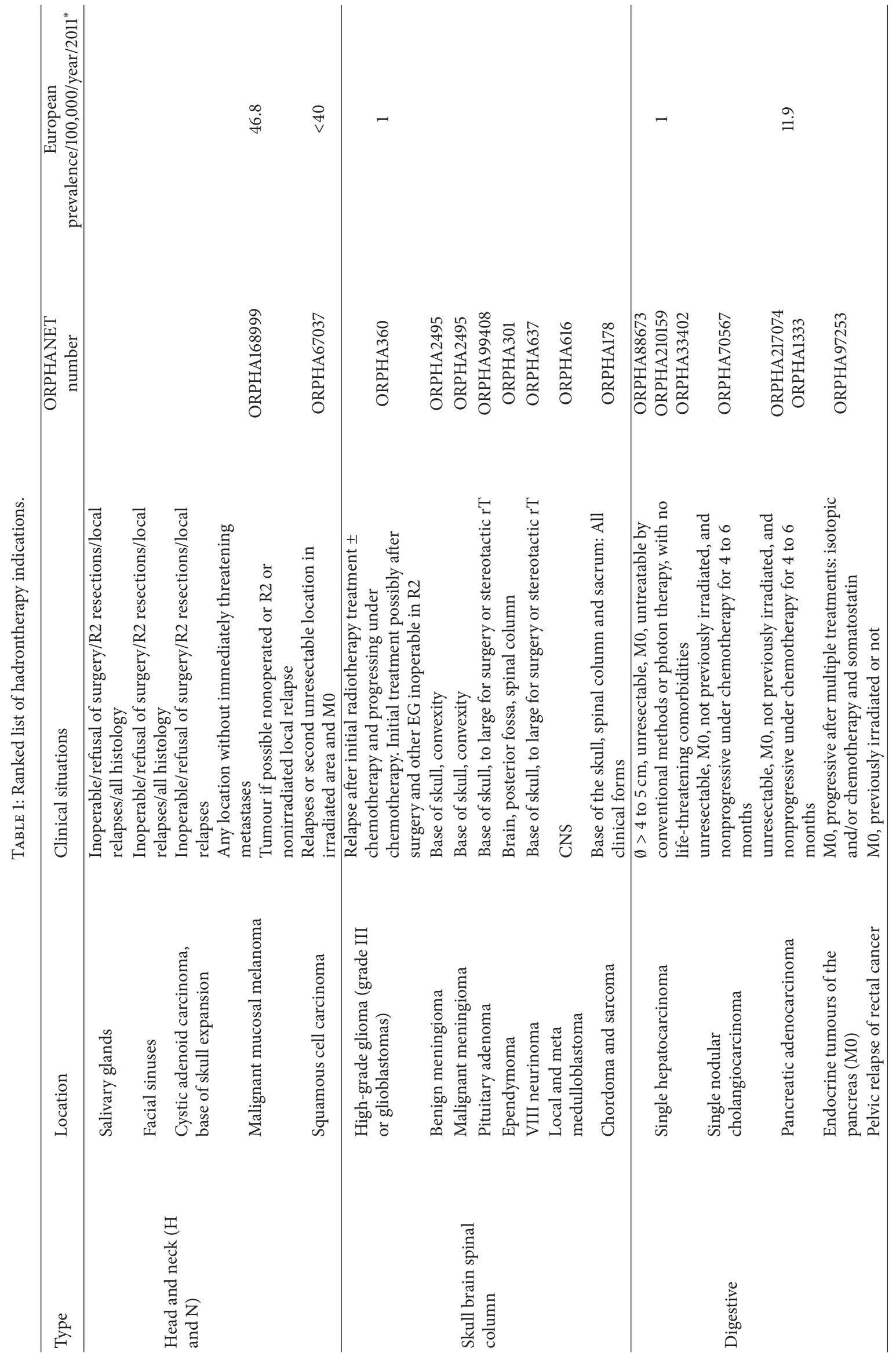




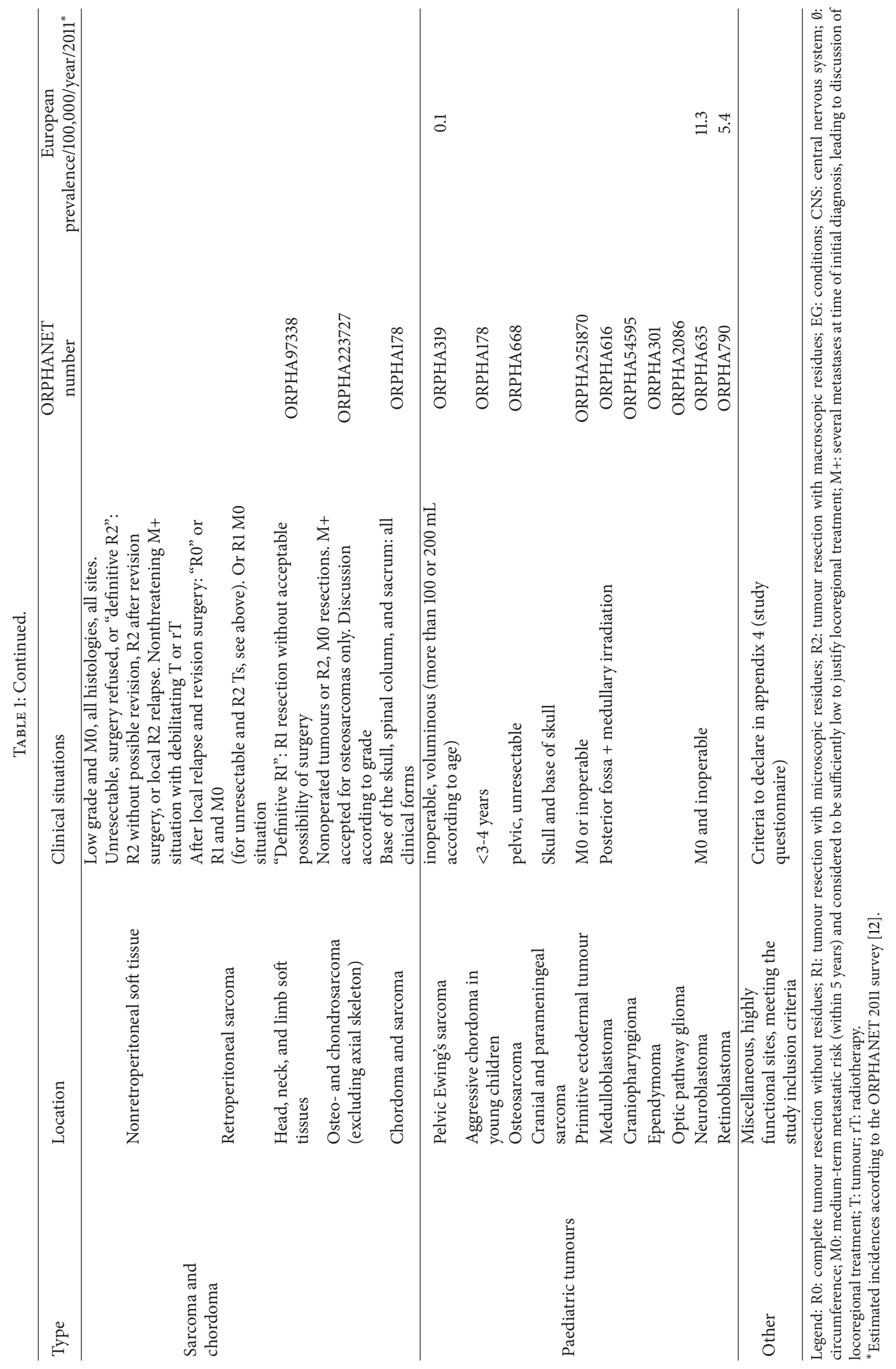


TABLE 2: Results, EpiHadron study: summary of incidence rates calculated during hadrontherapy epidemiological studies conducted between 1998 and 2004.

\begin{tabular}{|c|c|c|c|c|c|}
\hline Country & $\begin{array}{c}\text { Gross } \\
\text { incidence/year* }\end{array}$ & $\begin{array}{c}\text { Incidence/ } \\
/ 100,000 \\
\text { inhabitants/year* }\end{array}$ & $\begin{array}{l}\text { Total population } \\
\text { during the year of the } \\
\text { study (ù) }\end{array}$ & Study year & $\begin{array}{c}\text { Related healthcare } \\
\text { centre }\end{array}$ \\
\hline Italy & 10,825 & 19 & 56908265 & 1998 & CNAO \\
\hline Austria & 4873 & 61 & 7982461 & 1999 & Med-AUSTRON \\
\hline Switzerland & 263 & 4 & 7123537 & 1999 & Med-AUSTRON \\
\hline Slovenia (a) & 626 & 31 & 2000092 & 1999 & Med-AUSTRON \\
\hline Hungary & 153 & 1 & 10253400 & 1999 & Med-AUSTRON \\
\hline Italy & 313 & 1 & 56913634 & 1999 & Med-AUSTRON \\
\hline Czech Republic (b) & 5060 & 50 & 10190000 & 1999 & Med-AUSTRON \\
\hline Slovakia (y) & 1734 & 32 & 5477038 & 1999 & Med-AUSTRON \\
\hline France & 5320 & 9 & 61424036 & 2002 & ETOILE \\
\hline Austria & 2044 & 25 & 8063640 & 2002 & Med-AUSTRON \\
\hline Italy & 5579 & 10 & 57888245 & 2004 & CNAO \\
\hline
\end{tabular}

* Estimate expressed in study population, irrespective of disease location.

(y, a, b) Estimate of 2011, no data available before this date.

(ù) Demographic sources.

http://www.statistiques-mondiales.com.

http://appsso.eurostat.ec.europa.eu/nui/setupModifyTableLayout.do.

http://www.diplomatie.gouv.fr.

the tumours, clinical stage, pathology, therapeutic alternatives (e.g., surgical contraindications), and patient characteristics (general condition and comorbidities). French cancer registries record data required to estimate cancer incidence but fail to provide sufficient detail of the tumour stage or of therapeutic data. Moreover, the registries cover only approximately $15 \%$ of the French population and mainly for the most frequent cancers. Therefore, these registries do not allow the incidence of these tumours to be estimated.

1.5. Challenges and Prospects. This regional study will provide us with more detailed figures concerning the potential number of new cases eligible for hadrontherapy, allowing us to better adapt the future care offer delivered by the national hadrontherapy centre. This survey should also provide us with information on certain medical indicators to assist in the design of future hadrontherapy clinical trials (detailed incidence data, patient population characteristics, tumour stages, recruitment pools, etc.). Finally, this epidemiological field study is also eventually intended to be used to organise a network of highly specialised cares.

1.6. Hypothesis. According to the first epidemiological study carried out in France [6], in association with (i) the results of the literature review that defined the list of hadrontherapy indications and (ii) the indications chosen for proton therapy in France, the number of cases in the Rhône-Alpes region eligible for hadrontherapy is estimated at 200 per year (the Rhône-Alpes region is home to $10 \%$ of the French population).

\section{Goals}

The main goal is to asses, within the Rhône-Alpes region, the incidence of cancers with hadrontherapy indication.
The secondary goals are (1) to characterise the affected population, (2) to describe the characteristics of the observed cancers, and (3) to describe the characteristics of the treatments implemented when hadrontherapy is unavailable.

\section{Method}

Study Outline. This is a prospective, multicentre incidence study, lasting 24 months, conducted with the RCP (Réunion de Concertation Pluridisciplinaire-multidisciplinary tumor board) groups of the Rhône-Alpes region in France, that offer a therapeutic strategy for cancers for which hadrontherapy is an alternative.

Centre Recruitment and Inclusion Method. This study required the involvement of a part-time consultant epidemiology engineer, who totalled one woman-year of work to provide (1) phone canvassing to identify and involve regional cancer research players; (2) the protocol construction and testing phase, along with the data collection tools; (3) meetings with 3C (cancer coordination centres) coordinators and RCPs; (4) investigator centres inclusion meetings. An extensive number of travels within the region were required for these tasks.

Variables Collected. These included year of birth; gender; patient's department of residence; World Health Organization (WHO) performance status, patient's therapeutic status: initial stage or recurrence; initial staging (UICC TNM classification: International Union against Cancer); treatments received before recurrence; time to recurrence; staging at time of current tumour management; ICD-10 (International Classification of Diseases); ICDO (International Classification of Diseases for Oncology) histological type; site of surgery and margin quality; postsurgical histopathological stage (pTNM stage); prior radiotherapy; proposed treatment. 
"Patient" Inclusion Criteria. The criteria included adults and children, no age limit, and patients presenting with hadrontherapy indications listed in Table 1, whose medical files have been discussed by experts during an RCP. Subjects must be in compatible general and psychological condition.

Definition of "Patient" Inclusion Criteria. Compatible general and psychological condition refers to patients not suffering from life-threatening comorbidities (no acute or chronic diseases whose short-term lethal risk is dominant relative to the cancer), capable of adhering to a disease monitoring protocol and of understanding and accepting a complex treatment requiring close cooperation and staying for several days away from home.

"Tumour" Inclusion Criteria. These include unresectable tumour, belonging to a known radiation-resistant pathological group, mainly in a locoregional development stage, recurrent or local relapse, with low metastatic potential or $\mathrm{M}+$ dissemination (presence of one or more remote metastases at the time of diagnosis) presenting a low threat (slow development and/or metastases that can be readily treated). These inclusion criteria are the results of a consensus synthesis of a systematic literature review performed during 8 years, pathology by pathology with the participation of about one hundred of European medical, surgical, and radiation oncologists, managed by physicians trained in hadrontherapy (mainly protontherapy). The review was carried out by an academic laboratory specialized in clinical trial setup and analysis and was made according to the "evidence-based medicine" principle.

\section{Definition of "Tumour" Inclusion Criteria}

(i) Radiation resistance: tumour which estimated local dose required for local control that is higher that the maximum acceptable dose equivalent (MPDE) for surrounding organs necessarily irradiated under applicable technical conditions, assessed by a radiation oncologist (guide des Procédures de Radiothérapie Externe 2007 (guidelines for external radiotherapy procedures); joint effort by the French society for oncology radiotherapy (société Française de radiothérapie oncologique-SFRO), and the French society for medical physics (société française de physique médicale-SFPM), conducted in collaboration with the representatives of the French association of electroradiology paramedical workers (association française du personnel paramédical d'electroradiologie-AFPPE)).

(ii) Unresectable: tumour which local or locoregional expansion stage renders excision either is technically impossible (opinion of an experienced surgical team) or surgically unacceptable due to the irreparable damage that would be necessary (cancer: Principles \& Practice of Oncology 2005; DeVita, V. General articles on treatment strategies in cancer).

(iii) Locoregional: it refers to the tumour expansion state corresponding to the anatomical diffusion region of the primary tumour by contiguous expansion ( $\mathrm{T}$ of TNM) and/or by lymphatic diffusion, maintaining a rank of $\mathrm{N}$ in TNM staging, in other words, any tumour at stage M0 (latest edition of the tumour TNM classification published by the (UICC) International Union against Cancer or the (AJC) American joint committee for cancer staging).

(iv) Low metastatic potential: M0 situations whose medium-term (5-year) metastatic risk is considered to be sufficiently low to justify curative locoregional treatment involving significant means. This threshold is difficult to determine; a level greater than $50 \%$ seems unreasonable. As an example, mucosal melanomas are just at this threshold.

(v) Nonthreatening $M+$ dissemination or controlled by medical treatments: this means the presence of one or more remote metastases at the time of diagnosis, presenting no immediate threat as displaying slow development and/or accessible to effective treatment (typical case of some low-grade sarcomas lung metastases). These situations are indicative of locoregional disease treatment using complex and expensive techniques such as hadrontherapy.

(vi) Local relapse or local recurrence (these two terms are synonymous): it means the redevelopment, at the same site as initially, of a previously effectively treated tumour (dictionary of medical terms, Garnier-Delamare).

Primary Endpoint. it is the quantitative estimate of the incidence of indications eligible for hadrontherapy.

Secondary Endpoints. They are the description of the tumours and population characteristics, along with the treatments proposed and implemented by the RCPs.

Types of Investigation Centres and Participant. Those were healthcare establishments (private and public) in the RhôneAlpes region hosting RCP groups and having radiotherapy departments.

Types of RCP Groups Participating in Screening. they consisted of groups of oncology specialists with expertise in the following diseases: musculoskeletal sarcomas and tumours, Head and Neck ( $\mathrm{H}$ and $\mathrm{N})$, gastroenterology, paediatrics, dermatology, endocrine and central nervous system tumours.

Case Screening Modalities. Case screening is performed continuously by the physicians and clinical research technician in light of the list of indications (Table 1), during each RCP.

Data Collection Modalities. Data are collected in a decentralised prospective manner on paper questionnaires.

Study Monitoring. Fifteen percent of files were checked to ascertain data accuracy.

Study Quality. The questionnaire, along with the organisation of the investigation, was assessed beforehand by a sample 
population of investigators (3C coordinators and CRAs) via a semiguided telephone interview. For the questionnaire, the evaluation focused on general understanding, the form and length of the questionnaire, time required for completion, amount of data to collect, and the relevance of the selected criteria. The evaluation of the investigation method pertained to the choice of individuals involved in data collection organisation, the data recording method, the organisation for data recording, and the identification source for clinical cases.

Data Quality. Variables are encoded in a uniform and standard manner in an investigator's guide book, which contains detailed information concerning the definitions of each indicator to collect. Dual computer data input is performed, along with checks for missing or improbable data (encoding errors, date inconsistencies).

Statistical Methods. No sampling is performed as this study is intended to be exhaustive. The incidence of cancers is expressed as the number of cases reported from the demographic figures of the Rhône-Alpes region over the studied period and expressed per 100,000 inhabitants. Estimations of endpoint descriptive characteristics are expressed as frequencies, means, and percentages. Analyses are performed using Microsoft Excel, version 2008.

\section{Results}

The study involves 13 clinical research technicians, 50 RCP coordinating physicians and 12 cancer coordination centres (see Table 3). The study mobilises 27 Rhône-Alpes region healthcare centres, of which 8 university hospitals, 1 regional cancer centre, 1 public-private cancer institute, 12 general hospitals, the Lyon paediatric oncology hospital institute, and 4 private clinics. The study test phase validated the tools and organisation of this investigation. This led to a sense of ownership and motivation on the part of the centres. As a whole, the study covered the region's 11 hospital areas and involved 52 groups, out of some sixty listed, of cancer specialists with expertise in the diseases that were to identify.

During the study, 53 cases eligible for hadrontherapy were identified. Mean patient age was 43 years [2-89], and men were more numerous $(\mathrm{OR}=1.52)$. The highest number of cases $(39.6 \%)$ was in the $2-18$ years age group. The number of patients with recurrence was slightly lower (45.3\%) than the number of patients receiving initial care (54.7\%). Carcinomas, sarcomas (Table 4), and paediatric tumours (Table 5) were the most frequently identified diseases, and their locations are varied. During the RCP, radiotherapy, chemotherapy, and surgery were prescribed, respectively, in 58\%, 57\%, and $57 \%$ of cases. No brachytherapy was prescribed.

For the group of $45.3 \%$ relapsing patients, previous treatments were chemotherapy, surgery, or conventional radiotherapy in $62.5 \%, 58.3 \%$, and $58.3 \%$ of cases, respectively.

The number of cases identified during the study was approximately four times lower than the potential estimate. (Figure 2) Data collection was nonhomogeneous. The Isère department identified $43.4 \%$ of cases (i.e., 2.5 cases/RCP), and the Rhône department identified 56.6\% (i.e., 1.2 cases/RCP).
Isère identified twice as many cases as Rhône, even though it only has nine expert groups, that is, 2.6 times less than in Rhône, which has 24 . The geographic areas not covered by the study correspond to the location of 15 private healthcare centres and to the medical activity of six general and four specialist RCP groups. The first six groups had no recruitment potential according to the statement of their coordinators. The four other groups had a recruitment potential.

This preliminary data collection, conducted over a oneyear period, reported 53 cases of hadrontherapy indications, compared to the estimated cap of 200 cases. The Rhône-Alpes region was home to 6,222,045 inhabitants in 2010 [14]. The estimated incidence of cancers eligible for hadrontherapy in the Rhône-Alpes region in 2010, regardless of disease, is of $8.5 / 100,000$ inhabitants.

\section{Discussion}

5.1. About the Method. The diseases to identify in this study are little known, their distribution has not been studied, and we are unaware of any epidemic outbreaks. Moreover, considering that the cancers to identify are rare, it is likely that the number of cases to identify per investigator centre will be very low (estimated incidence of at the most 1 to 5 cases/year). Finally, the primary goal of the RCP groups is to provide an immediate therapeutic solution. Case identification for an observational study is thus unusual in the context of an RCP. Because of this, sustaining team vigilance and motivation for case identification is difficult to obtain and requires regular refreshing throughout the study.

The study test phase validated the tools and organisation chosen for this investigation. This step also served to circulate hadrontherapy information and to facilitate adhesion, ownership, and mobilisation around the project. The individuals involved in study organisation were in charge of systematically directing each cancer patient to the elective experts. Patients suffering from diseases eligible for hadrontherapy were frequently in relapse condition and rather at the end of their therapeutic options. This is due to the fact that these diseases have usually a slow and long evolution; therefore, this study had more chance to catch them somewhere in an advanced stage than at the beginning. It can also be considered that the lack of satisfactory treatment means as hadrontherapy makes these populations have more chance to show more advanced disease than it will be expected to be seen in the future. Their management requires discussion by a medical team of cancer specialists. The identification and decision to include a patient in the study were made by the RCP groups including these experts. Under these conditions, we have confidence in the case orientation and identification system.

The "tumour with low metastatic development potential" selection criterion is one of the criteria used to identify patients eligible for hadrontherapy. In our current state of knowledge, however, this indicator gives clinicians the greatest difficulties.

The results of this study were obtained mainly from public hospitals. This can be explained in part by the fact that it is easier to create multidisciplinary expert groups 
TABLE 3: Results, EpiHadron study: organisation, patient, and tumour characteristics.

\begin{tabular}{|c|c|c|}
\hline Categories & Population sizes $(n)$ & $\%$ \\
\hline \multicolumn{3}{|l|}{ Organisations } \\
\hline Investigating establishments & 27 & - \\
\hline RCP groups: multidisciplinary consultation meeting & 52 & - \\
\hline Cancer coordination centres & 12 & - \\
\hline RCP coordinating physicians & 50 & - \\
\hline Clinical research associate & 13 & - \\
\hline \multicolumn{3}{|l|}{ Patient characteristics } \\
\hline Patient age (mean) & 43 years $[2-89]$ & - \\
\hline Man & 32 & 60.4 \\
\hline Woman & 21 & 39.6 \\
\hline Initial care & 29 & 54.7 \\
\hline Recurrent cancer & 24 & 45.3 \\
\hline \multicolumn{3}{|l|}{ Tumour characteristics } \\
\hline Mean tumour size (largest circumference) & $69 \mathrm{~mm}[19-250]$ & - \\
\hline Metastases present & 11 & 20.8 \\
\hline Lymph nodes present & 10 & 18.9 \\
\hline \multicolumn{3}{|l|}{ Identified Cases } \\
\hline In total $(N)$ & 53 & - \\
\hline \multicolumn{3}{|l|}{ By age group } \\
\hline [2-18] years & 21 & 39.6 \\
\hline [18-45] years & 0 & 0 \\
\hline$[45-65]$ years & 18 & 34 \\
\hline [65-69] years & 0 & 0 \\
\hline [69-89] years & 14 & 26.4 \\
\hline \multicolumn{3}{|l|}{ By RCP type } \\
\hline Head and neck $\mathrm{H}$ and $\mathrm{N}$ & 4 & 7.5 \\
\hline Digestive & 12 & 22.6 \\
\hline Sarcoma and soft tissue & 2 & 3.8 \\
\hline Central nervous system CNS & 2 & 3.8 \\
\hline Paediatrics & 21 & 39.6 \\
\hline Dermatology & 4 & 7.5 \\
\hline General practice & 6 & 11.3 \\
\hline Oncology & 1 & 1.9 \\
\hline Other & 1 & 1.9 \\
\hline
\end{tabular}

in public hospitals and to set up clinical research. Due to their structure, these establishments benefit from human resources with more varied medical specialities than in private radiotherapy practices. In all likelihood, cases eligible for hadrontherapy could have been treated by these private centres during the study and ignored.

One should be very cautious when comparing the results of this study with those of published incidence studies. The methodologies employed in these previous studies are heterogeneous, and/or their flow is insufficiently documented. Moreover, their goal was to estimate a recruitment potential based on the extrapolation of the results. The methodologies used were not intended to provide information concerning an exhaustive incidence, as proposed by our study. Finally, the published studies were conducted in attractive institutions, specialising in cancer care and selected for this reason; this may bias the assessment of prevalence. Under these conditions, it is easy to confuse incidence and prevalence. It is thus conceivable that the estimation of incidence in the literature is overestimated. These elements could, in part, explain the observed difference between the preliminary results obtained in our study and the estimate calculated from the epidemiological results published in the literature. Only the publication of results obtained from other exhaustive epidemiological studies conducted in the same areas could support this hypothesis.

5.2. About the Results. It is unlikely that the lack of case recording associated with the medical activity of the four groups not involved in this data collection could alone explain the observed difference between the calculated incidence estimates and the preliminary results obtained. For several clinicians involved in the study, the prognosis and risk of metastatic development were difficult to estimate considering 
TABLE 4: Results, EpiHadron study: list of cases identified by anatomical type.

\begin{tabular}{|c|c|c|c|c|c|}
\hline Type & Category & Location & Population sizes $(n)$ & $\%$ & $\mathrm{C} / \mathrm{P}$ \\
\hline \multirow{13}{*}{ Carcinomas } & \multirow{4}{*}{ Adenocarcinoma } & of the endometrium & 1 & 1.9 & $\mathrm{C}$ \\
\hline & & of the pancreas & 1 & 1.9 & $\mathrm{C}$ \\
\hline & & of the rectum & 7 & 13.2 & $\mathrm{C}$ \\
\hline & & caecum with invasion of the psoas & 1 & 1.9 & $\mathrm{C}$ \\
\hline & \multirow{3}{*}{ Cystic glandular } & of the left parotid gland & 1 & 1.9 & $\mathrm{C}$ \\
\hline & & of the arytenoid cartilage & 1 & 1.9 & $\mathrm{C}$ \\
\hline & & of the scalp & 1 & 1.9 & $\mathrm{P}$ \\
\hline & \multirow{3}{*}{ Epidermoid } & oesophagus & 2 & 3.8 & $\mathrm{P}$ \\
\hline & & retromolar or intermaxillary commissure & 1 & 1.9 & $\mathrm{P}$ \\
\hline & & oropharynx & 1 & 1.9 & $\mathrm{P}$ \\
\hline & \multirow{3}{*}{ Other } & basal cell & 1 & 1.9 & $\mathrm{C}$ \\
\hline & & of the rectum & 1 & 2.3 & $\mathrm{C}$ \\
\hline & & neuroendocrine pancreatic & 1 & 1.9 & $\mathrm{C}$ \\
\hline \multirow[t]{5}{*}{$\begin{array}{l}\text { Total } \\
\text { carcinomas }\end{array}$} & & & 20 & 38.2 & \\
\hline & & of the humerus, upper end & 1 & 1.9 & $\mathrm{C}$ \\
\hline & Osteosarcoma & thigh and leg bone or cartilage & 1 & 1.9 & $\mathrm{C}$ \\
\hline & & osteogenic of the calvarium & 1 & 1.9 & $\mathrm{C}$ \\
\hline & Leiomyosarcoma & of the mucosal maxillary sinus & 1 & 1.9 & $\mathrm{C}$ \\
\hline \multirow[t]{6}{*}{ Sarcomas } & Chondrosarcoma & of the larynx & 1 & 1.9 & $\mathrm{P} / \mathrm{C}$ \\
\hline & Skin & posterior basicervical & 1 & 1.9 & $\mathrm{C}$ \\
\hline & Liposarcoma & & 1 & 1.9 & $\mathrm{C}$ \\
\hline & Ewing's & & 5 & 9.4 & $\mathrm{P} / \mathrm{C}$ \\
\hline & Rhabdoïd & clival & 3 & 5.7 & $\mathrm{C}$ \\
\hline & Grade II myxofibrosarcoma & antero-external part of the left leg & 1 & 1.9 & $\mathrm{C}$ \\
\hline \multirow[t]{4}{*}{ Total sarcomas } & & & 16 & 30.2 & \\
\hline & Neuroblastoma & of the base of the skull & 1 & 1.9 & $\mathrm{P}$ \\
\hline & & of the brain stem nuclei & 1 & 1.9 & $\mathrm{P} / \mathrm{C}$ \\
\hline & Glioma & of the optic nerve & 1 & 1.9 & $\mathrm{P}$ \\
\hline \multirow{6}{*}{$\begin{array}{l}\text { Skull Brain and } \\
\text { Spinal } \\
\text { column }\end{array}$} & & hypothalamic-chiasmatic & 1 & 1.9 & $\mathrm{P}$ \\
\hline & Glioblastoma & & 3 & 5.7 & $\mathrm{C}$ \\
\hline & Grade IV medulloblastoma & of the posterior cerebral fossa & 2 & 3.8 & $\mathrm{P}$ \\
\hline & Craniopharyngioma & & 1 & 1.9 & $\mathrm{C}$ \\
\hline & Malignant melanoma & of the left maxillary sinus & 1 & 1.9 & $\mathrm{C}$ \\
\hline & Xanthoastrocytoma & of the temporal lobe & 1 & 1.9 & $\mathrm{P} / \mathrm{C}$ \\
\hline \multirow[t]{2}{*}{ Total brain } & & & 12 & 22.6 & \\
\hline & Merkel cell tumour & of the left buttock & 1 & 1.9 & $\mathrm{C}$ \\
\hline \multirow[t]{2}{*}{ Other } & Chordoma & sacrum & 2 & 3.8 & $\mathrm{C}$ \\
\hline & Adrenal tumours & & 2 & 3.8 & $\mathrm{C}$ \\
\hline Total other & & & 5 & 9.4 & \\
\hline Totals $(N)$ & & & 53 & 100 & \\
\hline
\end{tabular}

Legendary: C: carbon, P: proton.

the lack of knowledge for these rare diseases. Moreover, the novelty of hadrontherapy, which can potentially modify usual treatment strategies, could have led to the omission of this indication in some cases, in particular conditions unusually treated by radiotherapy as hepatic and biliary tumours. These difficulties, along with the more sustained vigilance effort made by some groups relative to others, could account for the nonhomogeneous data collection and the observed difference between predictions and obtained results, despite the high motivation of the investigators. Furthermore, the 
TABLE 5: Results, EpiHadron: list of cases identified by anatomical type for paediatric tumours.

\begin{tabular}{|c|c|c|c|c|}
\hline Paediatric tumours & Category & Location & Population sizes $(n)$ & $\%$ \\
\hline \multirow{8}{*}{ Neurological tumours } & Neuroblastoma & The base of the skull & 1 & 4.8 \\
\hline & Neuroblastoma & Right adrenal gland & 1 & 4.8 \\
\hline & Neuroblastoma & The adrenal glands & 1 & 4.8 \\
\hline & Grade IV medulloblastoma & The posterior cerebral fossa & 2 & 9.5 \\
\hline & Glioma & The brain stem lymph nodes & 1 & 4.8 \\
\hline & Glioma & The optic nerve & 1 & 4.8 \\
\hline & Glioma & Hypothalamic-chiasmatic & 1 & 4.8 \\
\hline & Craniopharyngioma & & 1 & 4.8 \\
\hline \multirow{7}{*}{ Sarcomas } & Osteosarcoma & Thigh and leg bone and cartilage & 1 & 4.8 \\
\hline & Osteosarcoma & Upper end of the humerus & 1 & 4.8 \\
\hline & Osteogenic Osteosarcoma & The calvarium & 1 & 4.8 \\
\hline & Ewing's sarcoma & & 5 & 23.8 \\
\hline & Rhabdoid sarcoma & & 1 & 4.8 \\
\hline & Xanthoastrocytoma & Temporal neurological & 1 & 4.8 \\
\hline & Rhabdoid tumour & The clival region & 2 & 9.5 \\
\hline Total paediatric tumours & & & 21 & 100 \\
\hline
\end{tabular}

EpiHadron results

Reported cases per group involved per department
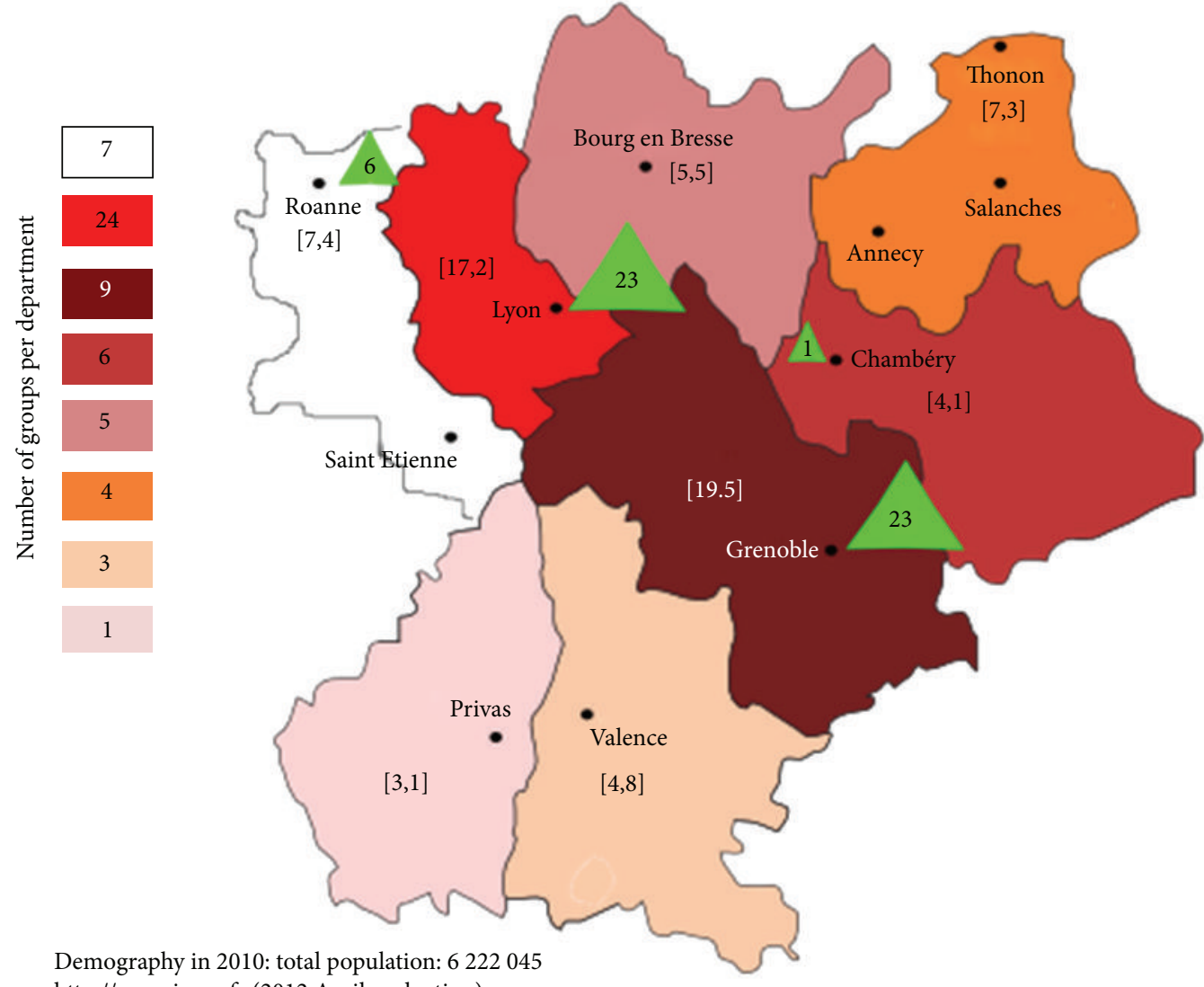

Demography in 2010: total population: 6222045 http://www.insee.fr (2012 April evaluation)

\section{Case number}

[] Total population per department,

in hundred thousands of inhabitants

FIGURE 2: Results, EpiHadron study: number of cases identified relative to the number of groups involved in each department. 
reorganisations (case of two major RCP clusters), changes of persons involved and the low frequency of cases to report, lead to a constant erosion of vigilance, requiring continuous action by the organiser.

This work aimed to structure a network of care pathway. However, some important institutions with their own recruitment targets did not wish to participate, which reduces the scope of our results.

About the detailed conditions of the recruited cases, there are a large proportion of recurrent diseases as explained above, and some metastatic diseases have also been included according to defined characteristics: (i) the number of metastasis was limited (1 to 3 metastasis); (ii) their very slow growing speed was not immediately threatening; (iii) the possibility of effective treatment of these "pauci-metastatic" conditions, essentially by ablative procedures. Actually, the principle to treat, with curative intend, patients in such situation is more and more accepted by expert oncologists.

The estimated incidence of cancers, irrespective of type and location, eligible for treatment by hadrontherapy, for the study, that is, $8.5 / 100,000$ individuals, is mildly higher than the reference incidence threshold of $\leq 6$ cases/100,000 individuals, as defined in the ORPHANET international database of "rare diseases" [12]. This figure can be obviously explained by the fact that the incidence was calculated by adding the five major cancer categories and 34 different locations. If one was to calculate the incidence for each of the disease types, their respective incidence would be significantly below the reference threshold of $\leq 6$ cases/100,000. Indeed, for our study, the minimum number of cases listed per disease is of 1 case and the maximum is of 7 cases. Table 4 .

Considering all of the difficulties and limitations of this preliminary phase of the study, the yield of approximately $25 \%$ notification, compared to the expected maximum, appears satisfactory. In the perspective of a (necessary) ramp up of a future hadrontherapy centre, there is thus a sufficient population to initiate operation and to progressively establish recruitment for this centre, without immediately generating an insufficient offer effect.

\section{Conclusion}

Future epidemiological studies will benefit from focusing on the characterisation of the metastatic development of diseases eligible for hadrontherapy. All cancers have their own development pattern, and, in this respect, the literature provides few elements concerning diseases eligible for hadrontherapy. This information should serve both to improve diagnosis procedures, medical care, and the surveillance of these rare diseases.

This study of hadrontherapy healthcare decision networks in the Rhône-Alpes region, able to identify areas not properly covered by RCPs, should enable us to analyse the potential effect of this lack on the outcome of the patients of these areas.

Incidence studies will be insufficient to determine the actual impact of hadrontherapy activity. These studies provide condensed elements about the medical activity associated with new patients only. They shed no light on posttreatment medical activity: follow-up visits, medical imaging, and other care beyond the irradiated activity per se.

One of the most valuable outcomes of this study has been to make the existence of some particularly rare diseases known and to provide information concerning an innovative treatment: hadrontherapy, whose existence will doubtless have a favourable effect on the very knowledge of these diseases. An additional benefit of this approach has been to open the way for a new treatment system in a region of France that is home to approximately $10 \%$ of the country's population. At last, these elective indications will have to be validated by health authorities to register them as part of the good professional practices of oncology and give an equal chance to each patient to receive hadrontherapy through the well-established decision process of the RCPs.

\section{Conflict of Interests}

The authors declare that they have no conflict of interests for this paper.

\section{Acknowledgments}

Warm thanks are due to the 27 care centers in the RhôneAlpes Region who participated: the 8 university hospitals of Lyon and Grenoble: Michallon Hospital, Women and Mother Child Hospital, Edouard Herriot Hospital, Cardiological Hospital Pierre Wertheimer, Neurological Hospital, Louis Pradel Hospital, Lyon-South Hospital Centers, and Croix Rousse Hospital; the 12 regional hospital centers: Thonon les Bains, Amberieux, Fleyriat, Villefranche, Pays de Giers, Roanne, Chambéry, Savoie Médipôle, Annecy, Sallanches, Valence, and Drôme North Hospital; the Institute of Haematology and Paediatric Oncology; and 3 clinics: Renaison, Marie Curie, and Praz Coutant; the regional center of fight against cancer Léon Bérard and the Cancer Institute Lucien Neuwirth of Saint-Etienne. The authors thank warmly, in particular, the $3 \mathrm{C}$ coordinators (Coordination Centers for Cancer): Dr. Leila Gofti-Laroche, cancer pole of the Inter-Hospital Oncology of the ARC ALPIN network: Isère, Savoies, Hautes Alpes, Ain, and Drôme; Dr. Jean Philipe Suchaud, Roanne Hospital, for Renaison Clinic and Roanne Hospital; Dr. Irène Philipe, Léon Bérard Center (CLB) of Lyon, for the Institute of Haematology and Paediatric Oncology and the CLB; Dr. Pierre Jean Souquet, Lyon South Hospital Centers; Dr. Maurice Perol, Croix Rousse Hospital, for Lyon North Hospitals: Hôtel Dieu Hospital, Livet Center, Dugoujon Geriatric Hospital, and Croix Rousse Hospital; Dr. Catherine Lombard-Bohas, Edouard Herriot Hospital (HEH), for Lyon-East Hospitals: Louis Pradel, Pierre Wertheimer, Women and Mother Child Hospital, and HEH; Dr. Hubert Orfeuvre, Fleyriat Hospital of Bourg en Bresse, for Ambérieu and Convert clinics, Haut-Bugey Hospital and Fleyriat Hospital. Alice Lacroix, Chambéry Hospital, for Savoie area: Médipole of Savoie, Hospital Centers of Aix les bains, Belley, Albertville Moutier, Bourg-Saint-Maurice, St. Jean de Maurienne, and the Chambéry Hospital; Dr. Jocelyne Provençal, Annecy Hospital, for South Savoie Hospitals: Praz 
Coutant, Sallanches, St. Julien, Leman Valserine, Argonay clinic, and Annecy Hospital; Dr. LI Véronique, Thonon les bains Hospital, for Leman Hospitals of North Savoie: Evian, Annemasse-Bonneville, and Thonon; Dr. Pierre Fournel, Cancer Institute of the Loire, for St. Etienne University Hopital, Hopitals of Annonay, Puy en Velay, Feurs, Pays de Giers, Montbrison, Firminy, Cévennes, Forez, the Parc clinics, network of hospitalization and health at home; Dr. Jean François Brantus, Villefranche Hospital for Tarare and Villefranche Hospitals; Dr. Robert Riou, Valence Hospital, for the following Hospitals: Valence, Crest, Die, Tournon, St. Marcellin, and for Pasteur and la Parisière clinics; Dr. Bernard Duvert, Montélimar Hospital, for Privas, Aubenas, and Montélimar Hospitals and for Vivarais and Kennedy clinics; Professor Jean Pierre Boissel, center of data treatment, UMR 5558, Medecine University of Laennec, Lyon, France. For the setup of this study, funding sources have been provided by the GCS ETOILE: http://centre-etoile.org/en/.

\section{References}

[1] H. Suit, "The gray lecture 2001: coming technical advances in radiation oncology," International Journal of Radiation Oncology Biology Physics, vol. 53, no. 4, pp. 798-809, 2002.

[2] H. Suit, S. Goldberg, A. Niemierko et al., "Proton beams to replace photon beams in radical dose treatments," Acta Oncologica, vol. 42, no. 8, pp. 800-808, 2003.

[3] R. Orecchia, M. Krengli, B. A. Jereczek-Fossa, S. Franzetti, and J. P. Gerard, "Clinical and research validity of hadrontherapy with ion beams," Critical Reviews in Oncology/Hematology, vol. 51, no. 2, pp. 81-90, 2004.

[4] K. Ando and Y. Kase, "Biological characteristics of carbon-ion therapy," International Journal of Radiation Biology, vol. 85, no. 9, pp. 715-728, 2009.

[5] R. Orecchia and M. Krengli, "Number of potential patients to be treated with proton therapy in Italy," Tumori, vol. 84, no. 2, pp. 205-208, 1998.

[6] H. Engels and A. Wambersie, "Cancer epidemiology and patient recruitment for hadrontherapy," Strahlentherapie und Onkologie, vol. 175, no. 2, supplement, pp. 95-99, 1999.

[7] M. H. Baron, P. Pommier, V. Favrel, G. Truc, J. Balosso, and J. Rochat, "A "one-day survey": as a reliable estimation of the potential recruitment for proton- and carbon-ion therapy in France," Radiotherapy and Oncology, vol. 73, supplement 2, pp. S15-S16, 2004.

[8] R. Mayer, U. Mock, R. Jäger et al., "Epidemiological aspects of hadron therapy: a prospective nationwide study of the Austrian project MedAustron and the Austrian Society of Radiooncology (OEGRO)," Radiotherapy and Oncology, vol. 73, supplement 2, pp. S24-S28, 2004.

[9] M. Krengli and R. Orecchia, "Medical aspects of the national centre for oncological hadrontherapy (CNAO-Centro Nazionale Adroterapia Oncologica) in Italy," Radiotherapy and Oncology, vol. 73, supplement 2, pp. S21-S23, 2004.

[10] T. Kamada, "Overview of the carbon ion radiotherapy at HIMAC," in Proceedings of NIRS-ETOILE 2nd Joint Symposium on Carbon Ion Radiotherapy, pp. 46-53, November 2011.

[11] International Classification of Rare Cancers, http://www.rarecare.eu/rare_indicators/WP5_Technical_Report.pdf.

[12] "ORPHANET Cahiers 2011: prevalence of rare diseases," http://www.orpha.net.
[13] H. Tsujii, J. Mizoe, T. Kamada et al., "Overview of clinical experiences on carbon ion radiotherapy at NIRS," Radiotherapy and Oncology, vol. 73, supplement 2, pp. S41-S49, 2004.

[14] "Estimated population by region, gender and high age-years 1990-2010," http://www.insee.fr. 


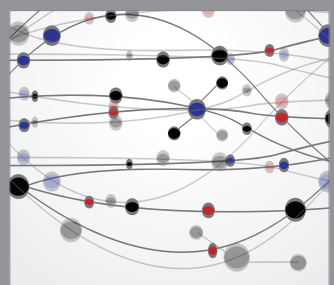

The Scientific World Journal
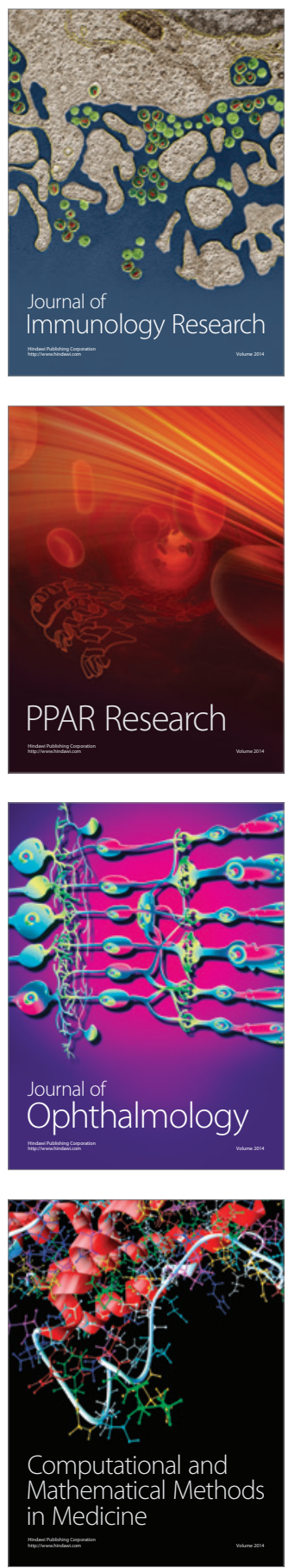

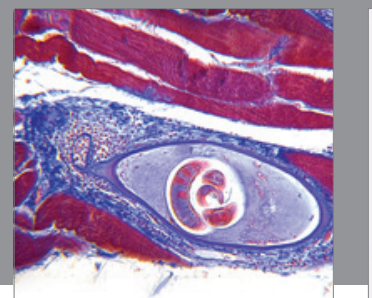

Gastroenterology

Research and Practice
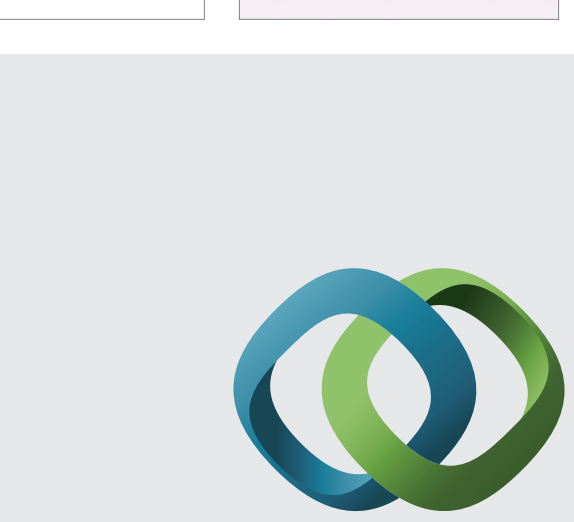

\section{Hindawi}

Submit your manuscripts at

http://www.hindawi.com
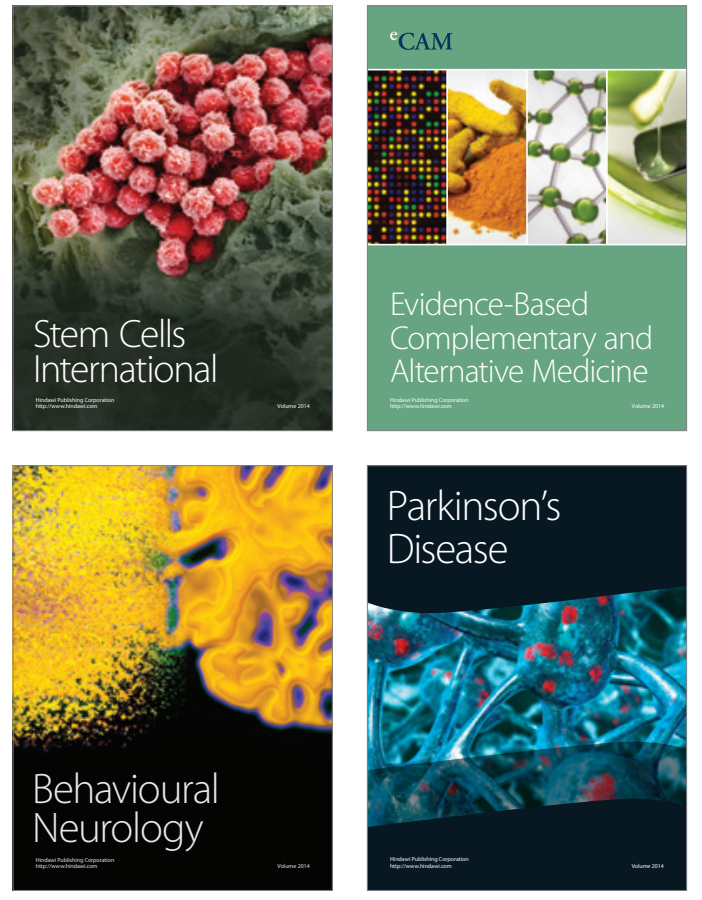
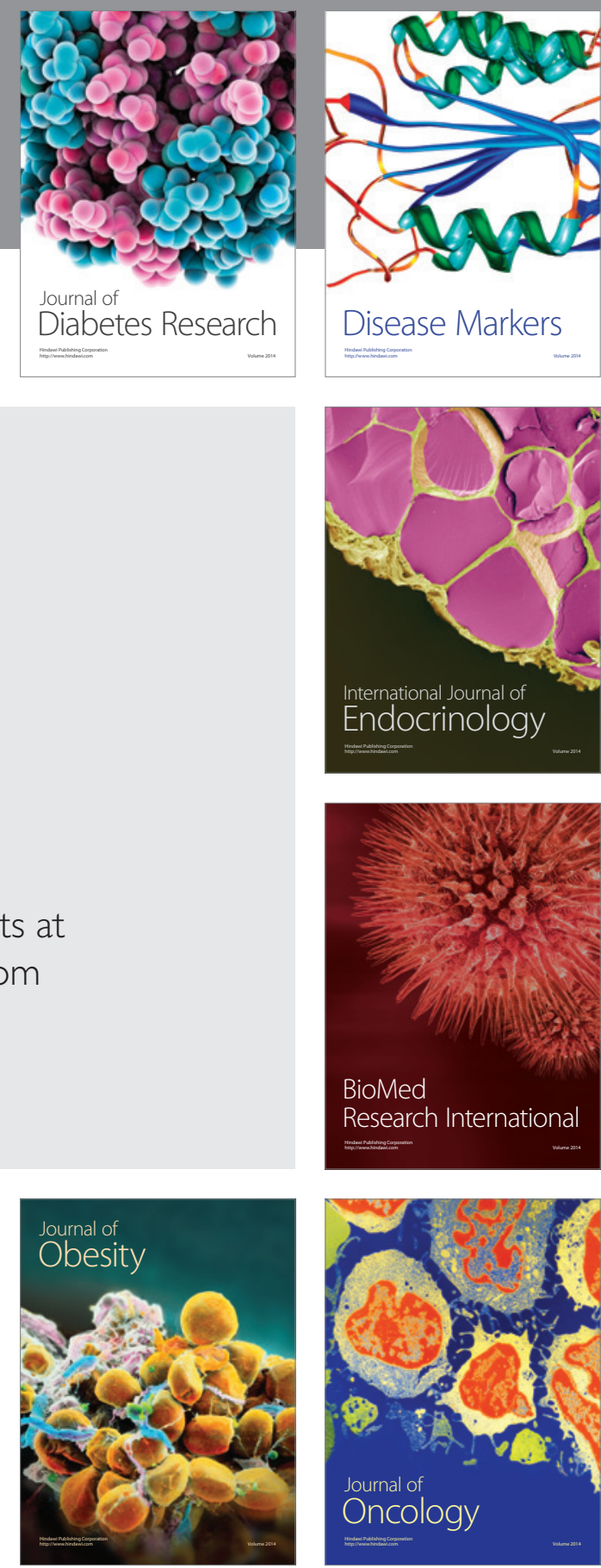

Disease Markers
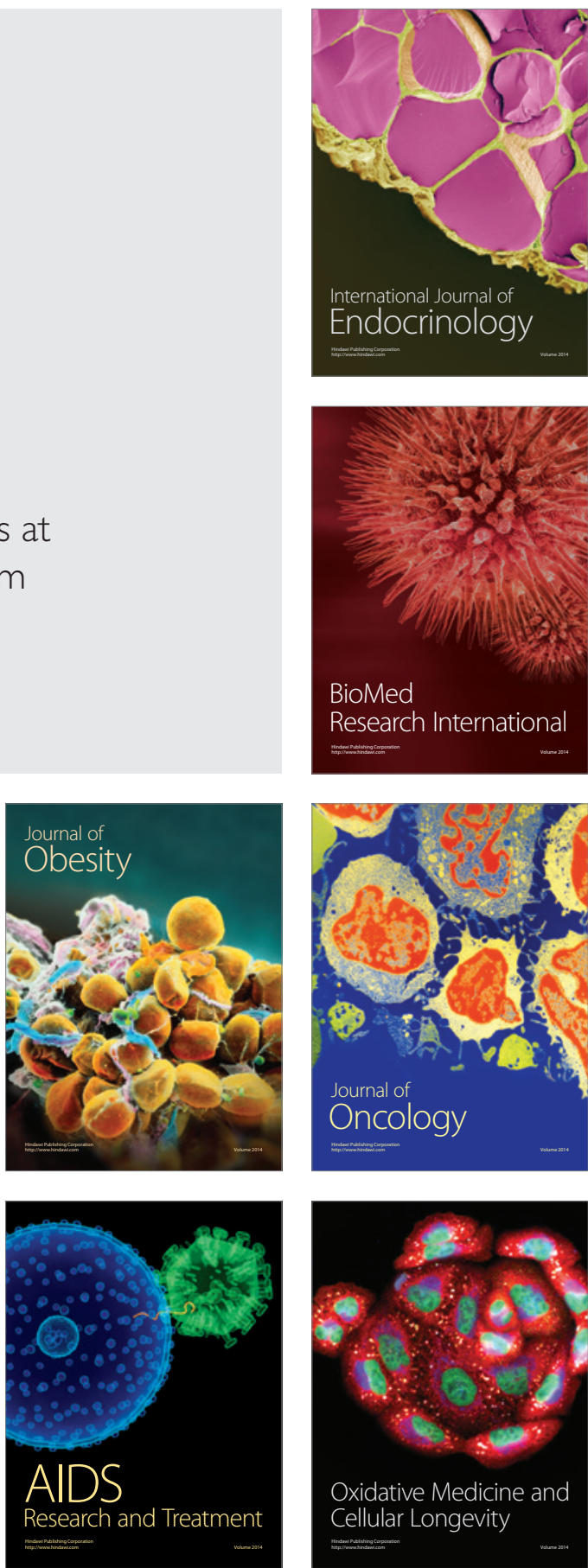